\title{
DE CLAUDE BERNARD A TELÉMACO BATTISTINI: LA MEDICINA EXPERIMENTAL EN EL NACIMIENTO DE LA REVISTA CIENTÍFICA DEL INSTITUTO NACIONAL DE SALUD DEL PERÚ
}

\author{
Franco Romaní1,2,a
}

\begin{abstract}
RESUMEN
La Revista Peruana de Medicina Experimental y Salud Pública (RPMESP) fue publicada por primera vez en 1942 con el nombre de Revista de Medicina Experimental, desde entonces ha sido el órgano de difusión científica del Instituto Nacional de Salud del Perú, el cual fue fundado años previos en 1936. En ambos acontecimientos históricos estuvo directamente involucrado Telémaco Battistini Sánchez. A partir de algunos pasajes de su historia personal y de la coyuntura histórica de la ciencia mundial y latinoamericana ocurrida entre 1865 y 1950, podemos comprender el porqué del uso del término «medicina experimental» en el nombre de dicha revista. El objetivo del artículo es describir una serie de eventos de la historia de la ciencia, que iniciaron en Europa y continuaron en el Perú, y que desencadenó la decisión de denominar como Revista de Medicina Experimental, a una revista que desde sus inicios ha publicado estudios observacionales, más aún en las últimas décadas, especialmente en el campo en el campo de la salud pública, mientras que las publicaciones de estudios en medicina experimental se han reducido drásticamente.
\end{abstract}

Palabras clave: Historia; Medicina experimental; Ciencia; Perú (fuente: DeCS BIREME).

\section{FROM CLAUDE BERNARD TO TELÉMACO BATTISTINI: EXPERIMENTAL MEDICINE IN THE BIRTH OF THE SCIENTIFIC JOURNAL OF THE PERUVIAN NATIONAL INSTITUTE OF HEALTH}

\begin{abstract}
The Peruvian Journal of Experimental Medicine and Public Health (RPMESP) was first published in 1942 under the name Journal of Experimental Medicine. Since then, it has been the official scientific dissemination vehicle of the Peruvian National Institute of Health, which was founded in 1936. Telémaco Battistini Sánchez was directly involved in both historical events. Based on some passages of his personal history and on the historical juncture of global and Latin American science between 1865 and 1950, we can understand why the term "experimental medicine" is used in the name of said journal. The aim of this article is to describe a series of events in the history of science which started off in Europe and continued in Peru and triggered the decision to give the name 'Journal of Experimental Medicine' to a journal that since its inception has published observational studies-more so in recent decades-, especially in the field of public health, while the number of publications of studies in experimental medicine has drastically decreased.
\end{abstract}

Keywords: History; Experimental medicine; Science; Peru (source: MeSH NLM).

\section{INTRODUCCIÓN}

Desde sus albores hasta la actualidad la Revista Peruana de Medicina Experimental y Salud Pública (RPMESP) ha publicado investigaciones en salud pública, por lo que su denominación actual, incluye el término «Salud Pública», pero también incluye un término que puede resultar menos familiar para los lectores, el de «medicina experimental». Con los cambios en la denominación de la revista se ha tratado de reflejar la producción científica en el país y su realidad sanitaria en diferentes épocas. $\mathrm{Si}$ bien, la historia de la RPMESP ha sido descrita en varias comunicaciones ${ }^{(1,2)}$, poco se ha discutido sobre la elección de su nombre, o sobre el contexto histórico científico que pudieron determinar dicha decisión. Sabemos que la ciencia es influenciada por factores históricos y sociales, y que la historia de la ciencia es en parte determinada por las historias de las personas, en este caso científicos.

\footnotetext{
Oficina General de Investigación y Transferencia Tecnológica, Instituto Nacional de Salud. Lima, Perú.

Universidad de Piura. Lima, Perú.

Médico cirujano

Recibido: 03/05/2019 Aprobado: 06/06/2019 En línea: 10/07/2019
}

Citar como: Romaní F. De Claude Bernard a Telémaco Battistini: la medicina experimental en el nacimiento de la revista científica del Instituto Nacional de Salud del Perú. Rev Peru Med Exp Salud Publica. 2019;36(3):530-4. doi:http://dx.doi.org/10.17843/rpmesp.2019.363.4512. 
En la actualidad, las revistas científicas son el principal instrumento de difusión de la ciencia, y como ella misma, no están exentas de las tendencias y hechos históricos que determinan paradigmas, corrientes y, por consiguiente, los nombres y denominaciones ${ }^{(3)}$.

Repasaremos eventos destacados de la historia de la ciencia inicialmente acontecidos en Europa, específicamente en Francia, con el nacimiento de la medicina experimental; las circunstancias de la introducción de ésta en Norteamérica; las acciones de la Fundación Rockefeller en América del Sur, incluyendo al Perú; y como un científico peruano formado en los Estados Unidos gracias a esta fundación, fue probablemente influenciado por la forma imperante de hacer investigación en dicha época; y que finalmente pudo generar la decisión de bautizar a la revista científica de su institución con el nombre de «Revista de Medicina Experimental».

El objetivo de esta publicación es comprender por qué una revista científica -que en la actualidad principalmente publica estudios observacionales- continúa incluyendo en su denominación el término «medicina experimental». Esto permitirá poner en valor el aporte de Claude Bernard a la medicina y ulteriormente a la salud pública moderna; y dilucidar que la medicina experimental y la salud pública no son términos excluyentes, sino afines.

\section{LA MEDICINA EXPERIMENTAL: SUS ORÍGENES EN FRANCIA Y SU DIFUSIÓN A AMÉRICA}

La idea de «la observación muestra y la experiencia instruye» fue acuñada para fortalecer la posición de que la experiencia es un privilegio de la razón. La historia ha demostrado que el hombre no se limita a ver, su naturaleza lo lleva a reflexionar, pensar y conocer los fenómenos cuya existencia le pudo haber revelado previamente la rigurosa observación. La comprobación por medio del razonamiento y de los hechos, constituye la experiencia, y según Claude Bernard, este es el único procedimiento que tenemos para instruirnos sobre la naturaleza de las cosas que están fuera de nosotros ${ }^{(4)}$.

Desde 1858, Bernard difundía esta línea de pensamiento, y venía trabajando en un Tratado de Medicina Experimental, en cuya primera parte plasmaría los principios de la Medicina Experimental. Dicha obra plasmó los preceptos a los cuales debería sujetarse la experimentación aplicada al estudio de los fenómenos de los seres vivientes. Introducción al Estudio de la Medicina Experimental fue publicada en 1865, mientras que en 1879 fue lanzada la obra Lecciones de Fisiología Operatoria, que representó la segunda parte que Bernard tenia proyectada para su Tratado de Medicina Experimental (4).
Para Bernard existían tres niveles de medicina: aquella basada en el «tacto y la intuición» considerada por él como la de más bajo nivel, la medicina "empírica» en el nivel intermedio y que esta basada en la comparación y la estadística, y la más avanzada la medicina "científica» basada en el descubrimiento experimental de las leyes o fenómenos de la naturaleza ${ }^{(5,6)}$.

La medicina experimental, según Bernard, es aquella que brinda el método científico para la medicina científica, y con ella se podría llegar al conocimiento de los mecanismos fisiológicos subyacentes a enfermedades específicas o al efecto terapéutico de una droga ${ }^{(5)}$. Bernard consideró a la fisiología y la medicina como ciencias progresivas y experimentales, cuyo adelanto debe buscarse con apoyo de la observación y el experimento, y con la ayuda del razonamiento experimental ${ }^{(4)}$.

Hoy sabemos que la medicina experimental tuvo gran influencia en el desarrollo de la fisiología y la medicina. La medicina experimental permitió vincular para siempre a la fisiología, la patología y la terapéutica. ${ }^{(4)}$ En su época Bernard visualizó la importancia de la evidencia científica en el conocimiento médico, sobre todo de aquella generada de los experimentos o ensayos comparativos; por ello ejecutó un sin número de experimentos con el que pudo contribuir a explicar fenómenos fisiológicos ${ }^{(5,6)}$.

Gracias en gran parte a Claude Bernard, en la segunda mitad del siglo XIX Francia jugó un rol primordial en el desarrollo de lo que posteriormente fue la medicina científica ${ }^{(7)}$, sin embargo, en las facultades de medicina francesas aún primaba el énfasis en anatomía y patología, las cuales eclipsaban a nuevas disciplinas como la bacteriología, y la «medicina experimental» y sus aplicaciones en estudios en fisiología. El trabajo de Bernard fue claramente más apreciado y aceptado en la comunidad científica extranjera, especialmente en Alemania y por la gran cantidad de estudiantes de medicina que llegaron a Francia provenientes de los Estados Unidos ${ }^{(8)}$.

En Alemania el desarrollo de la medicina científica siguió un rumbo distinto, Rudolf Virchow, que además de plantear la teoría celular y ser fundador de la medicina social, fue profesor de patología y fomentó la integración de la fisiología experimental en la educación médica. De esta forma, en las últimas décadas del siglo XIX y primera del $\mathrm{XX}$, los estudiantes norteamericanos prefirieron estudiar en las escuelas de medicina de Alemania ${ }^{(8)}$. En 1910 Abraham Flexner, educador formado en la Universidad Johns Hopkins en estudios clásicos y luego en la Universidad de Harvard en educación, visitó las principales escuelas de medicina en Gran Bretaña, Alemania y Francia. En su informe reforzó la idea ya conocida de la superioridad de la escuela alemana, y recalcó la importancia de la necesaria combinación de la enseñanza y la investigación tanto en el ámbito clínico (hospitales) como en los laboratorios ${ }^{(9,10)}$. El Informe de Flexner propuso una modernización de las escuelas de medicina en los Estados Unidos basada en la 
enseñanza de la medicina científica alemana y la tradición clínica de los franceses e ingleses. Dicha modernización fue patrocinada por la recientemente establecida Fundación Rockefeller, entidad que tuvo un rol fundamental en difundir la medicina científica (basada en la medicina experimental aporte primigenio de Bernard), así como su enseñanza, años más tarde, en las universidades de América del Sur. La triada de Flexner «sanar-enseñar-investigar» fue la base de la enseñanza médica en los Estados Unidos ${ }^{\left({ }^{8}\right)}$

\section{EL CONTEXTO LATINOAMERICANO Y EL NACIMIENTO DE LA REVISTA DE MEDICINA EXPERIMENTAL EN EL PERÚ}

Entre 1880 y 1950, algunos países de América del Sur crearon laboratorios en los cuales científicos locales, muchos de ellos formados en el extranjero, empezaron a desarrollar investigación experimental que empezó a ser reconocida a nivel internacional. El mayor reconocimiento fue logrado por investigadores en los campos de la bacteriología y fisiología, por ejemplo, en Brasil (Carlos Chagas), Argentina (Bernardo Houssay) y Perú (Carlos Monge). Estos investigadores y las instituciones a las cuales pertenecían emergieron en un periodo caracterizado por el nacionalismo, relativa prosperidad económica, e inicio del apoyo gubernamental a las actividades de ciencia y tecnología ${ }^{(8)}$

Entre 1913 y 1940, la Fundación Rockefeller (FR) empezó a financiar investigación en fisiología principalmente en Argentina y Perú. Los ejemplos más relevantes fueron las investigaciones en fisiología de la glándula pituitaria, páncreas, función renal y presión arterial llevadas a cabo por Bernardo Houssay en la Universidad de Buenos Aires; y los estudios de fisiología experimental para investigar los efectos de la altura en el humano liderados por Carlos Monge Medrano y Alberto Hurtado en el Instituto de Biología Andina de la Universidad Nacional Mayor de San Marcos. Posteriormente, el apoyo de la FR para la investigación fisiológica mediante la experimentación se extendió a México a partir de 1936, y Brasil a partir de $1946{ }^{(10)}$.

A partir de 1940, la educación médica en Latinoamérica se volvió una preocupación para la FR, quienes consideraban que las escuelas de medicina tenían un número excesivo de estudiantes en relación a sus instalaciones y profesores, elevado número de profesores de tiempo parcial, pobre desarrollo de las ciencias básicas, y sobre todo falta de trabajo en el laboratorio ${ }^{(10,11)}$. Estas características fueron atribuidas a la influencia de la escuela francesa en la enseñanza de la medicina clínica. Uno de los efectos observables de dicha escuela, era la falta de suficiente enseñanza de la fisiología experimental; lo cual propiciaba la práctica de la medicina empírica basada en la experiencia clínica y la observación. Los cursos de fisiología consistían en una repetición teórica, oral de los libros disponibles, con un fuerte componente de aprendizaje memorístico. La idea de realizar estudios experimentales para indagar acerca de la función de los órganos y del medio interno parecía ser un ejercicio innecesario para formación del futuro médico ${ }^{(10)}$.

Ante el panorama descrito, la FR implementó hasta cuatro estrategias: a) Brindar becas a estudiantes de medicina de América del Sur en las principales instituciones de investigación de los Estados Unidos, b) Promover la constitución de centros de educación médica y centros de investigación, c) Financiar investigación en ciencias básicas, y d) Donar equipos para investigación en fisiología experimental propiciando el trabajo de investigadores a tiempo completo ${ }^{(10)}$

Un grupo de jóvenes médicos peruanos recibieron becas de la FR para perfeccionar su formación en universidades norteamericanas. Entre estos se encontraba el futuro microbiólogo Telémaco Battistini Sánchez quien inició sus estudios de medicina en la Universidad Nacional Mayor de San Marcos, para luego de lograr el grado de médico en la Universidad de Madrid en 1921. Battistini obtuvo una beca de posgrado por la FR para sus estudios en bacteriología realizados entre 1924 y 1926 en la Universidad de Johns Hopkins y el Instituto de Investigación Médica Rockefeller ${ }^{(12)}$ donde colaboró con Hideyo Noguchi. Marcos Cueto destaca que la influencia decisiva de la formación en el exterior de los principales científicos peruanos de inicios del siglo $X X$, fue gracias a la interacción con científicos que aplicaban la medicina experimental en diversos campos, lo cual les permitió discernir entre una buena y mala investigación, y asimilar estándares de investigación para las instituciones peruanas ${ }^{(11)}$.

Battistini, diez años luego de su retorno al Perú en 1926, logró crear el Instituto Nacional de Higiene y Salud Pública (1936), el cual fue considerado una extensión del Instituto Rockefeller en el Perú. En 1942, el naciente instituto con el liderazgo de Battistini edita su revista científica, denominada «Revista de Medicina Experimental». La elección de dicho nombre obedecería al método científico predominante en dicho periodo, pues ya era extendido el uso de investigación experimental por la denominada élite científica limeña en campos como la bacteriología (Battistini, Barton, Gastiaburú, Hercelles) y la fisiología (Hurtado, Monge).

La publicación de la revista se mantuvo hasta 1960, luego hubo una brecha sin edición por más de tres décadas, hasta que en 1997 nuevamente fue publicada con la denominación de Revista de Medicina Experimental (Segunda época), en la editorial del número de relanzamiento se destacó el hecho que la revista reapareció en momentos en el que Perú, como muchos países latinoamericanos padecían la emergencia y reemergencia de enfermedades como el SIDA, el Cólera, la fiebre amarilla, dengue, tuberculosis resistente, entre otras ${ }^{(13)}$. En el 2002 toma la denominación actual como Revista Peruana de Medicina Experimental y Salud Pública. 


\section{EL ANTECEDENTE MÁS CERCANO, LA PROBABLE INSPIRACIÓN DE BATTISTINI}

En 1896, la revista científica denominada Journal of Experimental Medicine fue publicada en los Estados Unidos de Norteamérica y desde entonces ha publicado casi ininterrumpidamente 216 volúmenes hasta el 2019. El objetivo de esta revista, fundada 46 años antes de su contraparte peruana, fue publicar las investigaciones más destacadas en biología médica, y constituirse como una revista científica que integra los aportes de diversas disciplinas en el campo de la patogénesis. Actualmente como revista especializada en biología médica su foco temático son los estudios experimentales en humanos y modelos animales que indagan aspectos genéticos, inflamación, inmunidad, enfermedades infecciosas, cáncer, biología vascular, desordenes metabólicos, neurociencia y biología de células madre.

Esta revista fue fundada en el seno de la Escuela de Medicina de la Universidad de Johns Hopkins por William Welch, el fundador de la escuela de Medicina y también primer presidente del Cuerpo de Directores Científicos del Instituto Rockefeller. No fue hasta febrero de 1905 que el Instituto Rockefeller editó y publicó el primer número del Journal of Experimental Medicine con Simon Flexner (hermano Abraham Flexner) como editor. Desde dicho año la revista fue el principal instrumento de difusión científica de la investigación realizada por el Instituto Rockefeller, y de otros laboratorios externos. A la fecha la Universidad Rockefeller provee el acceso gratuito a texto completo a los artículos de esta revista.

Probablemente, Telémaco Battistini durante su estancia en la Universidad de Johns Hopkins y el Instituto Rockefeller, concluyó en la necesidad de una revista científica dedicada a la medicina experimental en el Perú, tomando como inspiración dicho nombre para la revista que años más tarde iniciaría (Figura 1).

\section{LO «EXPERIMENTAL»EN LA RPMESP}

Luego de su fundación y con la denominación de «Revista de Medicina Experimental» fueron publicados 30 números, los cuales publicaron artículos científicos en microbiología, fisiología, patología, farmacología o higiene. Un breve análisis de las características de los artículos publicados en la «Revista de Medicina Experimental» en sus tres primeros años, muestra que 40 artículos fueron publicados, de los cuales 36 correspondieron a investigaciones originales, de estas sólo $17(47,2 \%)$ fueron estudios que emplearon un diseño experimental. Entre los estudios experimentales, ocho fueron investigaciones farmacológicas, tres de infectología, dos de microbiología, uno de entomología y otro de fisiopatología, sólo dos correspondieron a investigaciones de fisiología experimental. Es decir, la «Revista de Medicina Experimental» desde sus inicios, publicaba estudios basado en observaciones, y por el ámbito de acción de su

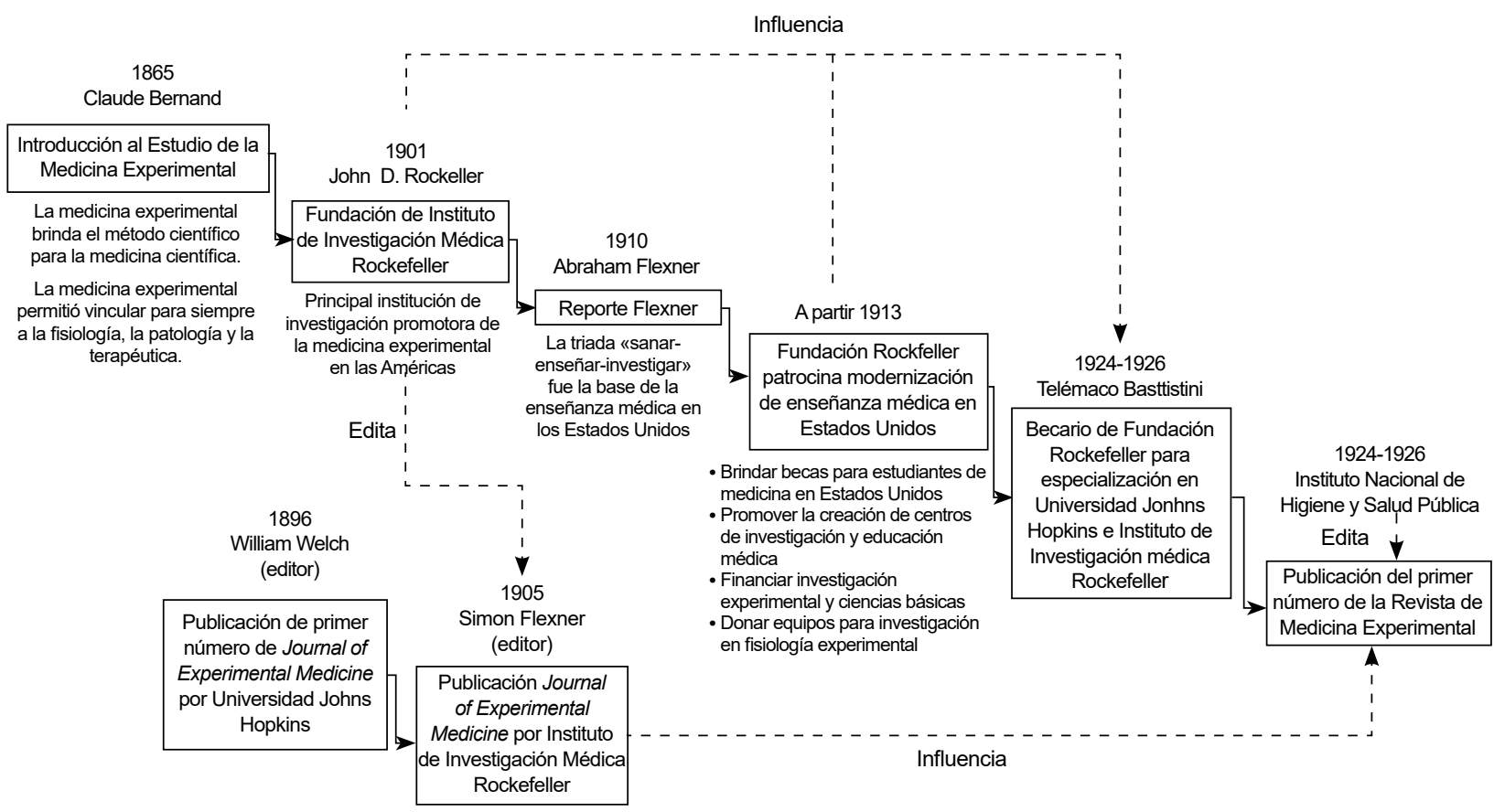

Figura 1. Actores e hitos de la historia de la ciencia destacados e influyentes en la denominación de la Revista de Medicina Experimental (1942) 
institución editora, estos estudios observacionales trataban mayormente temas vinculados a entomología, infectología, microbiología, y farmacología.

A pesar de lo descrito, según la editorial del primer número publicado ya con su denominación actual, la revista aceptaba desde 1997 artículos cuyo campo temático estaban vinculados a la salud pública en áreas de nutrición, salud ocupacional, salud ambiental, salud intercultural, políticas en salud, salud metal, investigación en servicios de salud (2). Sin embargo, podemos documentar que desde sus inicios la «Revista de Medicina Experimental» no se circunscribió exclusivamente a publicar estudios de fisiología o medicina experimental.

La preponderancia de publicaciones de estudios con diseño observacional en la RPMESP, se ha agudizado en la última década. Entre 2010 y 2017 , el 90,5\% de las investigaciones originales tuvieron un diseño observacional ${ }^{(14)}$. Sólo 38 estudios experimentales fueron publicados en dicho periodo, la mayoría de ellos fueron estudios para evaluar el efecto farmacológico de alguna sustancia derivada de especies vegetales.

\section{CONCLUSIONES}

Los acontecimientos históricos ocurridos en la comunidad científica peruana entre 1890 y 1930 definieron muchos hechos actuales, entre ellos, el nombre que adoptó la revista científica del Instituto Nacional de Salud. Lo ocurrido en el Perú no fue más que una adopción tardía de una corriente de pensamiento científico nacida en Francia en 1865, gracias a la contribución universal de Claude Bernard. La intervención de los Estados Unidos a través de la Fundación Rockefeller, catalizó la participación de la ciencia médica producida en América del Sur, en el movimiento científico mundial que primaba en la investigación científica biomédica (la medicina experimental). Es destacable que estos eventos históricos hayan ido progresando, de manera tal que terminaron influenciando en la historia personal de Telémaco Battistini y en esa coyuntura se encuentre la posible explicación del porqué en una revista que principalmente publica estudios observacionales, aún mantenga en su denominación el término «medicina experimental».

El nombre de nuestra revista debe ser visto como un recordatorio de la importancia que aún mantiene la medicina experimental, y que a pesar que cada vez recibimos menos manuscritos que desarrollan fisiología experimental o medicina experimental; consideramos dicha denominación como un tributo a Claude Bernard, así como a cada uno de los destacados médicos peruanos que entre 1880 y 1950 contribuyeron con su investigación experimental a hacer partícipe a la ciencia peruana del desarrollo científico mundial.

Agradecimientos: A los doctores César Cabezas Sánchez del Instituto Nacional de Salud, César Gutiérrez Villafuerte y Paolo Wong Chero de la Universidad de Piura por la revisión de manuscrito y sus valiosos comentarios a su contenido.

Contribución de autores: FRRR concibió la idea del artículo, analizó la información, redactó los borradores del manuscrito, y aprobó la versión final del manuscrito.

Fuente de financiamiento: Instituto Nacional de Salud.

Conflicto de interés: El autor se desempeña como Editor Científico de la Revista Peruana de Medicina Experimental y Salud Pública.

\section{REFERENCIAS BIBLIOGRÁFICAS}

1. Burstein Z, Cabezas C. 70 años de la Revista Peruana de Medicina Experimental y Salud Pública. Cumpliendo sus metas y proyectandose al futuro. Rev Peru Med Exp Salud Publica. 2012;29(1):6-8.

2. Editorial. Rev Peru Med Exp Salud Publica. 2002;19(1):3.

3. Casadevall A, Fang FC. (A)Historical Science. Bäumler AJ, editor. Infect Immun. 2015;83(12):4460-4.

4. Izquierdo JJ. Introducción al estudio de la medicina experimental. Versión castellana antecedida de una Historia crítica de su vida y sus trabajos. Segunda edición. Ciudad de México: Universidad Nacional Autónoma de México; 1960.

5. Morabia A. Claude Bernard was a 19th century proponent of medicine based on evidence. J Clin Epidemiol. 2006;59(11):1150-4. doi: 10.1016/j. jclinepi.2006.02.012.

6. Morabia A. Claude Bernard, statistics and comparative trials. J R
Soc Med. 2018;111(9):335-6. doi: $10.1177 / 0141076818796739$.

7. Ayala RA. Welcome to the New Age. Claude Bernard's "Introduction to the Study of Experimental Medicine" and the Shift of Medical Thought Towards Science: 150 Years Later. Arch Med Res. 2017;48(4):393-6. doi: 10.1016/j. arcmed.2017.08.006.

8. Schneider W. Rockefeller Philanthropy and Modern Biomedicine: International Initiatives from World Wwar I to the Cold War. Indiana: Indiana University Press; 2002.

9. Cueto M, Cañizares Esguerra I. History of Science in Non-Western Traditions: Latin America [Internet]. Notre Dame: History of Science Society [citado 23 abril de 2019]. Disponible en: https://hssonline.org/ resources/teaching/teaching_nonwestern/ teaching_nonwestern_latin/

10. Cueto M. The Rockefeller Foundation's Medical Policy and Scientific Research in Latin America: The Case of
Physiology. Social Studies of Science. 1990;20(2):229-25.

11. Cueto M. Excelencia Científica en la periferia. Actividades Científicas e Investigación Biomédica en el Perú. 18901950. Primera Edición. Lima:Grupo de Análisis para el Desarrollo GRADE; 1989.

12. Burstein Z. Telémaco Battistini Sánchez (1895-1960). Rev Peru Med Exp Salud Publica. 2010;27(1):142-5.

13. Editorial. Rev Med Exp INS. 1997;14(1):1.

14. Romaní F, Cabezas C. Indicadores bibliométricos de las publicaciones científicas de la Revista Peruana de Medicina Experimental y Salud Pública, 2010-2017. Rev Peru Med Exp Salud Pública.2018;35(4):620. doi: 10.17843/rpmesp.2018.354.3817.

Correspondencia: Franco Ronald Romani Romani

Dirección: Capac Yupanqui 1400, Jesús María. Lima, Perú.

Teléfono: 990630678

Correo electrónico:fromani@ins.gob.pe 